\title{
The scramble for influenza vaccine in 2010
}

In 2009 South Africa, like the rest of the world, experienced the 'swine flu' pandemic caused by influenza A (H1N1) 2009 virus (H1N1). The influenza epidemic curve for South Africa during 2009 testified to the introduction of the virus, as it superseded the influenza A H3N2 strain (H3N2) as the predominant circulating virus at the end of the season. ${ }^{1}$ Predicting patterns of influenza is difficult, and although most influenza cases in South Africa in 2010 may also be due to $\mathrm{H} 1 \mathrm{~N} 1$, it is uncertain to what degree H3N2 and influenza B will play their parts. Mercifully, H1N1 in 2009 caused mild influenza-like illness (ILI) in most infected persons; with 12640 laboratory-confirmed cases (a portion of the true number), only 93 laboratory-confirmed H1N1-associated deaths occurred., ${ }^{2,3}$

When a new pandemic influenza virus is introduced into a susceptible population, previously healthy people are at risk of severe disease, as are high-risk groups with underlying lung, heart, neurological or renal disease or those who are immunosuppressed either directly (e.g. HIV, transplant recipients) or relatively (e.g. pregnant women). In 2009 H1N1 did not seem to have the feared impact on the HIV-infected population, although data to judge this are incomplete. Of the 38 patients who died and had an HIV test, half were HIV infected. ${ }^{2}$ Owing to the overlap of clinical features between H1N1 and several HIV-related opportunistic infections, e.g. pneumocystis pneumonia, the true impact of H1N1 on HIV-related mortality remains unknown. Children were over-represented in terms of hospitalisation during 2009. The elderly, who are at increased risk during seasonal influenza epidemics, were not so affected, in part because of circulating levels of antibodies against previous H1N1 strains. ${ }^{4,5}$ Pregnant women experienced high morbidity and mortality, as occurred during the 1918 and subsequent epidemics. Those in the third trimester were particularly affected. ${ }^{6}$

During the 2009/2010 Northern Hemisphere influenza season, little antigenic drift occurred in circulating $\mathrm{H} 1 \mathrm{~N} 1^{7}$ and the clinical manifestations remain generally mild. In contrast, Southern Hemisphere H3N2 has undergone significant drift and could cause a large outbreak in high-risk groups such as the elderly in 2010 if it takes hold.

Events in 2009 emphasised the importance of protecting high-risk groups against contracting influenza by vaccination. A vaccine incorporating the pandemic H1N1 strain, H3N2, plus an influenza B strain was chosen for the 2010 Southern Hemisphere season. To prioritise receipt of vaccine in South Africa, the National Advisory Group on Immunization (NAGI) considered advice from the World Health Organization (WHO) Strategic Advisory Group of Experts (SAGE) ${ }^{8}$ and the Advisory Committee for Immunization Practices (ACIP). These suggested that all countries should immunise their health care workers (HCWs) as a first priority to protect the integrity of the health care system and reduce transmission, alongside consideration of reducing morbidity and mortality in highrisk groups. NAGI prioritised: ${ }^{9}$ first, pregnant women; second, persons $>6$ years of age in high-risk groups, including chronic lung, heart, neurological and renal disease, diabetes and related metabolic conditions, and persons on aspirin therapy; third, 'front-line' health and emergency personnel, i.e. doctors, nurses and emergency services personnel in casualty departments and 24-hour centres in direct contact with patients; and then HIV-infected adults, caregivers of infants, the elderly and the young.

Initially, 1.3 million doses of the trivalent vaccine were expected in installments, with the first 200000 by late February and 1.1 million by 12 April. Three million doses of Northern Hemisphere monovalent H1N1 vaccine were donated by the WHO. A significant delay in delivery of Southern Hemisphere trivalent vaccine was due partly to difficulties with growing stocks of the influenza B component. As the median start time of the influenza season in South Africa is the second week of June (range third week April - third week July), ${ }_{1}^{10}$ pressure mounted to procure the vaccine and start vaccinating those in need before the season started.

By the beginning of March, prioritisation changed to target four groups. Front-line HCWs were now prioritised first, followed by pregnant women, then HIV-infected children on antiretrovirals attending HIV clinics and patients with chronic lung and heart disease. These groups were divided into two phases of planned rollout, with HCWs and HIV-infected children $<15$ years first, followed by pregnant women and chronic lung and heart disease patients later. The day before the first phase was to begin, the Department of Health (DoH) directed that workers at ports of entry into South Africa were to receive vaccination first, to protect the integrity of these entry points. This prioritisation irrevocably linked the 2010 vaccination campaign in South Africa with that of keeping essential services operational for the FIFA World Cup. Further problems in obtaining the monovalent Northern Hemisphere H1N1 vaccine again delayed starting the campaign. This had been earmarked for HCWs, yet the delay meant that vaccination of HCWs using some of the trivalent vaccine stock was started. The prioritisation of supplies to the State sector led to minimal vaccine being available for private patients until late in the campaign, when vaccine was distributed through private pharmacies in June.

The prioritisation of HCWs to receive influenza vaccination in 2010 before high-risk groups raises several interesting questions.

In the context of the current pandemic caused by H1N1, was it correct to prioritise HCWs before giving vaccine to those most vulnerable in the population? The hypothesis is that by vaccinating front-line HCWs during a pandemic, the health system's ability to function is supported by reducing the number of key personnel who become ill, thus reducing absenteeism, safeguarding the ability of doctors and nurses to look after those infected, and reducing transmission of influenza to patients attending the health care facility. ${ }^{8}$ During the 2009 pandemic the South African health system maintained services although taking strain because of the increased number of staff and patients with symptoms. What 
is the evidence that in the second year after introduction of a pandemic virus that has caused low levels of disease activity during the Northern Hemisphere's 'second wave' in 2009 2010, the same strain on health services caused by H1N1 will occur in South Africa in 2010 as it did in 2009? Would port of entry staff have been prioritised had South Africa not hosted the FIFA World Cup? Why were the NAGI priorities changed in favour of HCWs? Where is the evidence to suggest that transmission of influenza from front-line staff to patients plays a significant role in the spread of infection in the population, over and above transmission in other settings? By vaccinating HCWs first, were we acting in the best interests of high-risk members of the population, whose risk of severe influenza and death is far greater than that of healthy doctors and nurses? A policy to ensure equity and prioritisation of the neediest in seasonal epidemics to come is of utmost importance.

How should we define a 'front-line' HCW in relation to receiving influenza vaccine? If the integrity of a health system that also relies on other health professions is threatened, should only doctors and nurses in emergency units and intensive care units be targeted? If pharmacists, radiographers or other key personnel were absent, how would the hospitals cope? A future more inclusive approach must be considered, even if supplies to each group of HCWs are limited, so that at least a proportion of staff in each profession is covered.

Prioritising front-line staff hampers our ability to protect high-risk patients by withholding vaccination from HCWs who work in units with high-risk patients, e.g. obstetric and transplant units, HIV clinics, and outpatient clinics caring for those with chronic lung, heart, renal and kidney disease, and diabetics. Targeting front-line staff may provide more 'bang for our buck', but at the expense of equity and protecting those known to get more severe disease.

By concentrating on HCWs and groups that were at greatest risk during the 2009 pandemic, the 2010 vaccination campaign puts the elderly on a lower priority, although we know that they have the highest risk of severe disease and death during seasonal epidemics. ${ }^{11}$ If predictions that H1N1 will play the major role in the South African epidemic are wrong and H3N2 predominates or contributes significantly, the elderly will have a potentially high morbidity and mortality.

How do we ensure future increased vaccine uptake, as poor uptake is a major challenge to effective protection of those in need, internationally and in South Africa? ${ }^{12,13}$ How will prioritisation of HCWs in 2010 be seen by high-risk groups, and will it detract from the urgency of ensuring improved uptake? A concerted educational campaign to improve uptake for future influenza seasonal epidemics is required. There is unlikely to be a better time to drive home this public health message than now, when the influenza threat is fresh in the mind.

The question is not whether HCWs should be excluded from receiving influenza vaccination, but to what extent they should have been prioritised before high-risk groups. In a utopian world we would have had enough vaccine to cover the entire population in need, well before the influenza season started. Unavoidable delays compounded a difficult situation. Prioritising in a resource-limited setting is an invidious task. Yet to see to the needs of the most vulnerable in the population, bearing in mind that most previously healthy people will experience no symptoms or mild ILI, we must scrutinise how we prioritise the future use of influenza vaccine and debate the issue further.

The author has no conflicting interests to declare.

\section{Marc Mendelson}

Division of Infectious Diseases and HIV Medicine

Department of Medicine

Groote Schuur Hospital and

University of Cape Town

Corresponding author: M Mendelson (marc.mendelson@uct.ac.za)

References

1. National Institute for Communicable Diseases. Situation update pandemic influenza A(H1N1) 2009-2010 South Africa. 24 April 2010. http://www.nicd.ac.za (accessed 14 May 2010).

2. National Institute for Communicable Diseases. Influenza A/H1N1 ('Swine Flu'). Updated 15 February 2010. http:// www.nicd.ac.za (accessed 14 June 2010).

3. Archer BN, Cohen C, Naidoo D, et al. Interim report on pandemic H1N1 influenza virus infections in South Africa, April to October 2009: Epidemiology and factors associated with fatal cases. Euro Surveill 2009; 14(42): pii 19369 .

4. Ikonen N, Strengell M, Kinnunen L, et al. High frequency of cross-reacting antibodies agains 2009 pandemic influenza A(H1N1) virus among the elderly in Finland. Euro Surveill 2010; 15(5): pii $=19478$.

5. Miller E, Hoschler K, Hardelid P, Stanford E, Andrews N, Zambon M. Incidence of 2009 pandemic influenza A H1N1 infection in England: a cross-sectional serological study. Lancet 2010; 375: 1100-1108.

6. Langenegger E, Coetzee A, Jacobs S, le Roux A, Theron G. Severe acute respiratory infection with influenza A (H1N1) during pregnancy. S Afr Med J 2009; 99(10): 713-716.

7. Johansen K, Nicoli A, Clanciao BC, Kramarz P. Pandemic influenza A (H1N1) 2009 vaccines in the European Union. Euro Surveill 2009; 14(41): pii=19361.

8. World Health Organization. Strategic Advisory Group of Experts on Immunization - report of the extraordinary meeting on the influenza A (H1N1) 2009 pandemic, 7 July 2009. Wkly Epidemiol Rec 2009; 84(30): 301-308.

9. Department of Health. Recommendations pertaining to the use of viral vaccines: Influenza 2010. S Afr Med J 2010; 100(2): 88-89.

10. McAnerney JM, Blumberg L. Active influenza surveillance in South Africa. Presented at the Conference of the Federation of Infectious Diseases Societies of Southern Africa, 26 July 2005, Sun City, North West, South Africa. Poster ID P29.

11. Karstaedt AS, Hopley M, Wong M, Crewe-Brown HH, Tasset-Tisseau A. Influenza- and respiratory syncitial virus-associated adult mortality in Soweto. S Afr Med J 2009; 99: 750-754

12. Keenan H, Campbell J, Evans PH. Influenza vaccination in patients with asthma: Why is uptake so low? Br J Gen Pract 2007; 57: 359-63.

13. Van Vuuren A, Rheeder P, Hak E. Effectiveness of influenza vaccination in the elderly in South Africa. Epidemiol Infect 2009; 137: 994-1002. 